\title{
Treatment of Bruxism with Trihexiphenidyl, a Case Series
}

\author{
Seyyed Mohammad Moosavi ${ }^{1}$, Javad Setareh ${ }^{2}$, Mahshid Ahmadi ${ }^{3}$, Mani B. Monajemi ${ }^{4,}$, \\ Soudabeh Shafiee Ardestani ${ }^{5}$
}

${ }^{1}$ Mazandaran University of Medical Sciences, Department of Psychiatry, Sari, Iran

${ }^{2}$ Mazandaran University of Medical Sciences, Sari, Iran

${ }^{3}$ Mazandaran University of Medical Sciences, Department of Social Medicine, Sari, Iran

${ }^{4}$ University of Tehran, Department of Clinical Psychology, Tehran, Iran

${ }^{5}$ Tehran University of Medical Sciences, Department of Medicine, Tehran, Iran

Email address:

mani.b.monajemi@warwickgrad.net (M. B. Monajemi),mani.b.monajemi@ut.ac.ir (M. B.Monajemi)

\section{To cite this article:}

Seyyed Mohammad Moosavi, Javad Setareh, Mahshid Ahmadi, Mani B. Monajemi, Soudabeh Shafiee Ardestani. Treatment of Bruxism with Trihexiphenidyl, a Case Series. American Journal of Psychiatry and Neuroscience. Vol. 3, No. 6, 2015, pp. 108-110.

doi: 10.11648/j.ajpn.20150306.12

\begin{abstract}
Bruxism is a sleep related movement disorder defined by repetitive jaw muscle activity that causes clenching or grinding of the teeth. Bruxism can lead to teeth and jaw damage and disturbs sleeping process. Although gastro-esophageal reflux, smoking and disturbances in CNS dopaminergic system implicated in etiology of bruxism but no clear etiology is defined. There are plenty of treatments were used for these patients included biofeedback, hypnosis, occlusal splints, beside drugs. Pharmacological treatments such as Clonidine, Clonazepam, Hydroxizine, Sodium valproate, selective serotonin reuptake inhibitors (SSRIs) sometimes induce bruxism. Case reports showed that SSRIs induced bruxism resolved by Buspirone but tricyclic antidepressants were not supported to be effective treatment for bruxism in papers. It is hypothesized that the direct and indirect pathways of the basal ganglia are involved in the coordination of movements is disturbed in bruxer like in Parkinson disease. Based on these facts we had thought Trihexyphenidyl may be effective in resistant patients. This study describes 4 patients whom suffered from bruxism which resisted to usual treatments. Trihexiphenidyl (Artane) an anticholinergic drug reduced symptoms in all cases. Anticholinergic drugs can be considered for treating bruxism. Both night and day types of bruxism, as a movement disorder was relieved in response to Thp, probably due to anticholinergic effects in basal ganglia. These isolated cases could not be generalized. Further placebo controlled clinical trials are needed to evaluate and proof the efficacy of anticholinergic drugs such as Thp for treating of bruxism.
\end{abstract}

Keywords: Anticholinergic, Bruxism Treatment, Trihexiphenidyl

\section{Introduction}

Bruxism is a sleep related movement disorder defined by repetitive jaw muscle activity that causes clenching or grinding of the teeth. It can be categorized to night and daily movements (1). The results of bruxism may be discomfort and/or damage of teeth, Tempromandibular pain, headache, cosmetic problems and also associated with hypertension (23 ). 14 to 38 percent of children and $8-31.6 \%$ of adults were reported to have Bruxism and mean prevalence of sleep bruxism was $12.8 \%$ (4-5). Although gastro-esophageal reflux, smoking and disturbances in CNS dopaminergic system implicated in etiology of bruxism but no clear etiology is defined (6-7). Many treatments were used for these patients included biofeedback, hypnosis, occlusal splints, beside drugs (8-9). Pharmacological treatments such as Clonidine, Clonazepam, Hydroxizine, Sodum valproate (10-11) Selective seretonine reuptake inhibitors (SSRIs) sometimes induce bruxism. Case reports showed that SSRIs induced bruxism resolved by Buspirone (12). Amitriptyline and other tricyclic antidepressants were not supported to be effective treatment for bruxism in papers (13). Botulinum toxin was used for treatment of bruxism and sometimes with serious adverse effects (14).

AntiParkinson drugs which have dopaminergic effects may relief and antidopaminergic drugs can exacerbate bruxism. Bruxism may be a Prodromal feature of Parkinson disease (15). It is hypothesized that the direct and indirect pathways of the basal ganglia are involved in the coordination of movements is disturbed in bruxer like in Parkinson disease. 
Based on these facts we had thought Thp may be effective in bruxism.

After explanation about the study to each of participants and reassurance about being anonymous, written informed consent was obtained from all participants before initiation of the study.

In this article 4 cases with bruxism who were treated with Trihexiphenidyl successfully reported.

Case 1:

The patient was a 17 year old boy referred with night bruxism. He suffered almost every night for 3 years. The most disturbing was its loud sound of greeting that leaded to night time awakening. He didn't use any drug such as metoclopramide, he was not smoker, did not use substances and also he had not history of dental problems as his dentist reported. The patient had no positive finding in past medical history and physical and neurological examinations, except for recent dental problems consequence the disorder. Except mild anxiety, no obvious mental disorder was seen in psychiatric interview. He reported that his father had untreated bruxism frequently for a couple of years. Previous medication such as Amitriptyline, Nortriptyline and Propranolol was not effective as patient and parents' report. Clonazepam $0.5 \mathrm{mg}$ per day had moderate effects for reduction of symptoms but he discontinued it because of daily drowsiness. Trihexyphenydil $0.5 \mathrm{mg}$, 2hour before sleep was administrated. One week later he reported his bruxism reduced moderately .Trihexiphenidyl (Thp) increased to $1 \mathrm{mg}$ every night and in the next week and no symptom of bruxism was reported later. After 45th day the dosage of Thp decreased to $0.5 \mathrm{mg}$ and after 30 days discontinued. Patient was symptom- free for 11 nights and after that bruxism relapsed and gradually became as severe as before treatment. Thp 1mg started again and bruxism disappeared rapidly.

Case 2:

The patient was 27 year old man referred with night and daytime bruxism from 4 month ago with wax and wane in daily to weekly periods. He had repetitive clenching of jaw leading to pain at tempromandibular joints. He did not use any drug and no history for medical, psychiatric disorders and substance abuse or dependency prior to bruxism. No dental occlusion disorder was reported in dentist consultation. Also symptoms started 3 month after loss of his father, he was not diagnosed as depressive disorder and pathological grief in psychiatric interview. The symptoms had not relieved by using anxiolytics (Clonazepam) or tricyclics (Imipramine) each for 2 weeks period. Thp $1 \mathrm{mg}$ divided in 2 times prescribed. Symptoms relieved for 3 days after treatment and continued for 60 days but relapsed 1 week after discontinuation. Thp prescribed again and symptoms reduction was prominent.

Case 3:

A 22 year old female university student referred by gastroenterologist to private psychiatric clinic. She had night bruxism since early adolescence. Investigations did not show any medical, neurological and substance use disorder. Although the patient reported that the symptoms exacerbated under stress but symptoms never removed more than 2-3 days even without stressors. No drug was used from 2 years ago and she refused dental guard because of its discomfort. She used to recieve Propranolol, Diazepam, Alprazolam, Amitriptyline, Doxepine, H1 Blocker (cetrizine) without positive effects. Thp prescribed for this patient and the effects were like case no 1 .

Case 4:

The patient was 16 year old female, high school student. She had bruxism almost every night. She had also jaw and dental pain, but the major reason of refer was family complaint of her sound of teeth gridding. Symptoms had been started when she was a child with wax and wane course, exacerbated under stress but never removed more than 1 month and had been severe in recent 3 months before referral. No drug, no medical and/or psychiatric disorder were reported in history of patient except for night terror in childhood. She had positive family history (father had bruxism). Her grandfather suffered from Parkinson disease. Physical and neurological examinations showed no positive finding. Benzodiazepines (diazepam) had no effect in 3 weeks trial, but imipramine was effective slightly. Thp $1 \mathrm{mg}$ each night prescribed and symptoms removed in third night totally. The patient was symptom free for 3 months. Symptoms relapsed 1 week after discontinuation of thp. Symptoms relieved 3 days after Thp restarted with the same dose.

\section{Discussion}

Although the pathogenesis of bruxism remains unclear, bruxism episodes were associated with arousal response along with involuntary leg movements. It is hypothesized that the direct and indirect pathways of the basal ganglia are involved in the coordination of movements is disturbed in bruxer like in Parkinson disease (15). Sleep Bruxism is associated to rhythmic masticatory muscle activity characterized by co-activation of both jaw-opening and jawclosing muscles instead of the alternating jaw-opening and jaw-closing muscle activity pattern typical of chewing. This is also like Parkinson disease in which both agonist and antagonist muscles co-activity are present. Acute use of dopamine precursors like L-dopa inhibits bruxism activity and chronic long term use of L-dopa results in increased bruxism activity (16).

All 4 patients were suffered from repetitive clinching of jaw, 3 in night and 1 on day, medical problem related to bruxism were excluded and no definite relationship to drug and substance usage were found. All of patients tried other routine drugs for bruxism and no relief was found among them. Symptoms were disappeared rapidly by Thp, an anticholinergic drug. Symptoms relapsed soon after discontinuation and removed by restarting of Thp. This low dose of Thp $(1-2 \mathrm{mg} / \mathrm{d})$ has minimal side effects comparing to benzodiazepines, antihistamines, alpha ${ }_{2}$ adrenergic agonists (clonidine), or invasive methods such as botulism injections $(11,16)$. Thp has been used in Parkinson disease and acute 
and chronic dystonia both categorized as movement disorder. Bruxism also is considered a movement disorder. The effect of anticholinergic drugs in dystonia and Parkinson disease is mediated via cholinergic synapses of basal ganglia so the pathogenesis of bruxism may be the same as above mentioned disorders.

The novel point in this article was an effort to find out a treatment of bruxism. As mentioned above variety of pharmacological and non- pharmacological issues have been offered to treat this suffering condition but most of them were not effective or the effectiveness was weak or temporary. Trihexyphenidyl use supports with the semi- clear pathogenesis of bruxism but had not considered previous researches. This clinical trial may be the first step to attention to it and may open a window to try these types of drugs to treat the disorder in a larger sample-size, placebo- controlled investigation in future.

\section{Conclusion}

The current paper presents an experimental model for treatment of resistant bruxism. In this study both night and day types of bruxism, as a movement disorder was relieved in response to Trihexyphenidyl, probably due to anticholinergic effects in basal ganglia. Our study was conducted on only 4 patients with resistant bruxism and these few isolated cases could not be generalized. Further placebo controlled clinical trials are needed to evaluate and proof the efficacy of anticholinergic drugs such as Trihexyphenidyl for treating of bruxism.

\section{Authors' Contributions}

SMM and JS conceived and designed the evaluation. MBM collected clinical data and conducted statistical evaluation. SSA and MA interpreted the clinical data and drafted the manuscript. All authors read and approved the final manuscript.

\section{Acknowledgements}

We would like to thank all patients who participated in the study.

\section{References}

[1] Lobbezoo, F., et al., Bruxism defined and graded: an international consensus. J Oral Rehabil, 2013. 40(1): 2-4 .doi: 10.1111/joor.12011. PMID: 23121262.

[2] Carra, M.C., et al., Sleep bruxism, snoring, and headaches in adolescents: short-term effects of a mandibular advancement appliance. Sleep Med, 2013. 14(7):656-61.doi: 10.1016/j.sleep.2013.03.009. PMID: 23643652.

[3] Fernandes, G., et al., Temporomandibular disorders, sleep bruxism, and primary headaches are mutually associated. J Orofac Pain, 2013. 27(1):14-20. doi: 10.11607/jop.921. PMID: 23424716

[4] Cheifetz, A.T., et al., Prevalence of bruxism and associated correlates in children as reported by parents. J Dent Child (Chic), 2005. 72(2):67-73. PMCID: PMC3446016.

[5] Manfredini, D., et al., Epidemiology of bruxism in adults: a systematic review of the literature. J Orofac Pain, 2013. 27(2):99-110. doi:10.11607/jop.921.

[6] Feu, D., et al., A systematic review of etiological and risk factors associated with bruxism. J Orthod, 2013. 40(2):63.71doi: 10.1179/1465313312Y.0000000021. PMID: 23794697.

[7] Chen, W.H., et al., A proposed mechanism for diurnal/nocturnal bruxism: hypersensitivity of presynaptic dopamine receptors in the frontal lobe. J Clin Neurosci, 2005. 12(2):161-3 .PMID: 15749418.

[8] Ilovar, S., et al., Biofeedback for treatment of awake and sleep bruxism in adults: systematic review protocol. Syst Rev, 2014. 3:42. doi: 10.1186/2046-4053-3-42.

[9] Dowd, E.T., Nocturnal bruxism and hypnotherapy: a case study. Int J Clin Exp Hypn, 2013. 61(2):205-18. doi: 10.1080/00207144.2013.753832. PMID: 23427844.

[10] Macedo, C.R., et al., Pharmacotherapy for sleep bruxism. Cochrane Database Syst Rev, 2014. 10:Cd005578. doi: 10.1002/14651858.CD005578.pub2. PMID: 25338726.

[11] Huynh, N.T., et al., Comparison of various treatments for sleep bruxism using determinants of number needed to treat and effect size. Int J Prosthodont, 2006. 19(5):435-41. PMID: 17323720 .

[12] Sabuncuoglu, O., O. Ekinci, and M. Berkem, Fluoxetineinduced sleep bruxism in an adolescent treated with buspirone: a case report. Spec Care Dentist, 2009. 29(5):215-7.doi: 10.1111/j.1754-4505.2009.00091.x. PMID: 19740153.

[13] Raigrodski, A.J., et al., The effect of four-week administration of amitriptyline on sleep bruxism. A double-blind crossover clinical study. Cranio, 2001. 19(1):21-5.PMID: 11842836.

[14] Webster, J.B., C.E. Poorman, and D.X. Cifu, Guest editorial: Botulinum toxin injection for bruxism associated with brain injury: case report. J Rehabil Res Dev, 2014. 51(4):vii-xvi .doi: 10.1682/JRRD.2013.10.0218. PMID: 25144179.

[15] Srivastava, T., et al., Bruxism as presenting feature of Parkinson's disease .J Assoc Physicians India, 2002. 50:457. PMID: 11922248.

[16] Lavigne, G.J., et al., Neurobiological mechanisms involved in sleep bruxism. Crit Rev Oral Biol Med, 2003. 14(1):30-46. PMID: 12764018. 